\title{
Profil évolutif et pronostic des tumeurs urothéliales de la vessie chez le sujet jeune
}

\author{
Abderrazak Bouzouita, MD; Ahmed Saadi, MD; Walid Kerkeni, MD; Marouene Chakroun, MD; \\ Mohamed Cherif, MD; Haroun Ayed, MD; Selim Selmi, MD; Amine Derouiche, MD; \\ Riadh Mohamed Benslama, MD; Mohamed Chebil, MD
}

Service d'urologie, hôpital Charles-Nicolle,Tunis, Tunisie

Cite as: Can Urol Assoc J 2016;10(3-4):E1 10-3. http://dx.doi.org/10.5489/cuai.3298

\section{Résumé}

But : Les tumeurs urothéliales de la vessie sont rares chez le jeune adulte. Leur profil évolutif et leur pronostic restent matière à controverse. Nous rapportons notre expérience à propos de 54 patients. Méthodologie : Entre 1990 et 2010, 54 patients de moins de 40 ans au moment du diagnostic ont été traités pour carcinome à cellules transitionnelles de la vessie. Nous avons étudié le profil évolutif de ces tumeurs en séparant les patients en deux groupes (moins de 30 ans, et 30 à 40 ans).

Résultats : La tumeur n'infiltrait pas le muscle vésical dans 37 cas et l'infiltrait dans 17 cas. Pour les tumeurs n'infiltrant pas le muscle vésical, elles étaient de stade Ta dans 20 cas et de grade I-II dans 36 cas. Le pronostic de ces tumeurs était meilleur avant I'âge de 30 ans avec un taux de récidive de 15,3\% sans progression. Pour les patients de 30 à 40 ans, le taux de récidive était de 33,3\%, et $25 \%$ des tumeurs qui ont récidivé ont présenté une progression du stade. Pour les tumeurs infiltrant le muscle vésical, le pronostic était sombre (localement avancées dans neuf cas et métastatiques d'emblée dans cinq cas).

Conclusion : Le profil évolutif des tumeurs n'infiltrant pas le muscle vésical a semblé meilleur avant l'âge de 30 ans. Entre 30 et 40 ans, le profil évolutif s'est approché de celui des sujets âgés. Les tumeurs infiltrantes étaient souvent évoluées et agressives, évoquant un potentiel évolutif particulier.

\section{Introduction}

Les tumeurs de la vessie surviennent généralement chez le sujet âgé entre 50 et $70 \mathrm{ans}^{1,2,3}$.

L'atteinte chez le jeune adulte (moins de 40 ans) est rare et ne dépasse pas $4 \%$ selon les séries ${ }^{1,2,4-7}$. L'évolution naturelle et le pronostic du carcinome à cellules transitionnelles (CCT) de la vessie chez les jeunes patients restent un sujet de débat et peu de données ont été publiées concernant cette entité. De plus, les quelques études ayant analysé le pronostic du cancer de la vessie dans cette population ont abouti à des résultats contradictoires. Certains auteurs considèrent que 30 ans est un âge charnière au-delà duquel I'agressivité de la tumeur urothéliale de la vessie rejoint celle du sujet âgé, et en deçà duquel le pronostic est meilleur ${ }^{1,3,6-9}$.

Dans cette étude, on s'est proposé de déterminer le profil évolutif et le pronostic des tumeurs urothéliales de la vessie chez le jeune adulte.

\section{Méthodologie}

Il s'agit d'une étude rétrospective, descriptive et monocentrique, étalée sur une période de 20 ans, soit de 1990 à 2010, ayant inclus 54 patients de moins de 40 ans au moment du diagnostic et porteurs de tumeur urothéliale de la vessie. Le diagnostic a été confirmé par examen histologique après résection endoscopique de la tumeur ou après cystectomie. Seuls les carcinomes à cellules transitionnelles ont été inclus dans notre étude. Nos patients ont été séparés en deux groupes : le groupe I comprend les patients âgés de moins de 30 ans et le groupe II comprend les patients âgés de 30 à 40 ans.

\section{Résultats}

Il s'agissait de 50 hommes et 4 femmes. L'âge moyen était de 34 ans (15 à 40 ans). L'hématurie macroscopique était le signe d'appel dans tous les cas. Des signes d'irritation vésicale ont été notés dans 28 cas (51,85\%). Un tabagisme a été noté dans 30 cas $(55,5 \%)$ et une exposition professionnelle dans cinq cas $(9,25 \%)$.

\section{Résultats globaux}

Sur l'ensemble des 54 patients ayant une tumeur urothéliale, $37(68,5 \%)$ avaient une tumeur $n^{\prime}$ infiltrant pas le muscle vésical (TVNIM) et $17(31,5 \%)$ avaient une tumeur infiltrant le muscle vésical (TVIM). 
Les TVNIM étaient de stade Ta dans 20 cas et T1 dans 17 cas. Dix-huit tumeurs étaient de grade I, 18 tumeurs étaient de grade II et une seule tumeur était de grade III. La tumeur était multifocale ( $\geq 3$ foyers) dans huit cas et volumineuse $(>3 \mathrm{~cm})$ dans quatre cas. Tous les patients ont eu une résection endoscopique complète, suivie d'une immunothérapie endovésicale à base de BCG dans 19 cas. Aucune instillation de mitomycine n'a été réalisée. Une récidive tumorale a été notée dans 10 cas; elle est survenue chez les patients porteurs d'une tumeur Ta dans quatre cas et T1 dans six cas. La tumeur initiale était multifocale dans cing cas et volumineuse dans deux cas. Le délai moyen de récidive était de 3 ans. Deux tumeurs ont récidivé au cours du traitement par BCG. Parmi les patients qui ont présenté une récidive tumorale, une progression du stade a été notée dans deux cas; la tumeur initiale était multifocale, de stade T1 et de grade II dans les deux cas et elle est devenue une TVIM après la récidive (Tableau 1). Le premier patient a eu une cystoprostatectomie totale avec bonne évolution (recul de 4 ans), le deuxième a eu une radiochimiothérapie palliative devant une récidive massive et inopérable (Tableau 1).

Pour les TVIM, elles étaient localement avancées dans dix cas et métastatiques d'emblée dans cinq cas. On a réalisé une cystoprostatectomie totale dans dix cas avec une survie de $50 \%$ à 5 ans. Dans les sept cas restants, le traitement était palliatif et tous les patients sont décédés dans un délai moyen de 10 mois (6-18 mois).

\section{Résultats en fonction de l'âge}

\section{Groupe $1(<30$ ans) : 15 cas}

La tumeur était une TVNIM dans 13 cas (86,6\%), de stade Ta dans huit cas et T1 dans cinq cas. La majorité des tumeurs étaient bien différenciées : grade I dans neuf cas, grade II dans quatre cas et aucun cas de grade III. La tumeur était monofocale dans 12 cas et bifocale dans un cas. Une seule tumeur était volumineuse. Six patients ont eu une BCGthérapie complémentaire. Deux patients (15,3\%) ont présenté une récidive sans progression quant au stade ou au grade. Ces deux récidives sont survenues après un délai de

\begin{tabular}{|c|c|c|}
\hline$N=37$ & Nombre & Pourcentage \\
\hline T1 & 17 & $45,9 \%$ \\
\hline Grade II-III & 19 & $51,3 \%$ \\
\hline Multifocale ( $\geq 3$ foyers) & 8 & $21,6 \%$ \\
\hline Volumineuse $(>3 \mathrm{~cm})$ & 4 & $10,8 \%$ \\
\hline Récidive & 10 & $27 \%$ \\
\hline Progression & 2 & $5,4 \%$ \\
\hline
\end{tabular}

3 et 4 ans, respectivement, pour des tumeurs initiales de stade T1 et de grade II.

La tumeur était une TVIM dans deux cas. Dans un cas, on a réalisé une cystoprostatectomie totale avec dérivation urinaire externe de type Bricker. Le patient est décédé après 18 mois après une altération de son état général avec apparition de métastases osseuses. Dans le deuxième cas, on a réalisé une cystoprostatectomie totale avec entérocystoplastie de remplacement. Les suites étaient favorables avec un recul actuel de sept ans.

\section{Groupe II ( $30-40$ ans) : 39 cas}

La tumeur était une TVNIM dans 24 cas $(61,5 \%)$ : de stade Ta dans 12 cas et T1 dans 12 cas. Elle était de grade I dans neuf cas, de grade II dans 14 cas et de grade III dans un cas. La tumeur était multifocale dans huit cas et volumineuse dans trois cas.

Treize patients ont eu une BCG-thérapie. Huit patients $(33,3 \%)$ ont présenté une récidive tumorale avec progression du stade dans deux cas (25\%) (Tableau 2).

La tumeur était une TVIM dans 15 cas. Toutes les tumeurs localement avancées (dix cas) et métastatiques (cinq cas) appartiennent à ce groupe. Sept tumeurs étaient jugées inopérables et traitées de façon palliative. Huit patients ont eu un traitement chirurgical à visée curative avec une survie à 5 ans de $50 \%$ (Tableau 3).

\section{Discussion}

Le CCT de la vessie survient essentiellement à partir de la cinquantaine $e^{1-3,5}$. Avant 40 ans, cette pathologie est rare et ne dépasse pas $4 \%$ selon les séries ${ }^{1,2,4-7}$. Selon McCarty, la fréquence du CCT de la vessie avant 30 ans est estimée à $0,8 \%{ }^{10}$. La prédominance masculine est nette dans toutes les séries $1,3,4,5,7,11$. Les mêmes facteurs de risque décrits chez le sujet âgé sont retrouvés chez le jeune adulte ${ }^{1,4,5}$. Il est cependant difficile chez le sujet jeune, notamment avant 30 ans, d'affirmer le rôle de l'exposition au tabac ou aux carcinogènes professionnels (peinture, solvant...), compte tenu de la faible durée d'exposition'. Plusieurs auteurs évoquent

\begin{tabular}{|c|c|c|}
\hline & $\begin{array}{c}\text { Groupe } 1 \text { (<30 ans): } \\
n=13\end{array}$ & $\begin{array}{c}\text { Groupe II (30-40 } \\
\text { ans): } n=24\end{array}$ \\
\hline T1 & $5(38,4 \%)$ & $12(50 \%)$ \\
\hline Grade II-III & $4(30,7 \%)$ & $15(62,5 \%)$ \\
\hline Multifocale ( $\geq 3$ foyers) & 0 & $8(33,3 \%)$ \\
\hline Volumineuse ( $>3 \mathrm{~cm}$ ) & $1(7,6 \%)$ & $3(12,5 \%)$ \\
\hline Récidive & $2(15,3 \%)$ & $8(33,3 \%)$ \\
\hline Progression & 0 & $2(8,3 \%)$ \\
\hline
\end{tabular}




\begin{tabular}{|c|c|c|}
\hline & $\begin{array}{c}\text { Groupe } 1 \text { ( }<30 \text { ans): } \\
n=2\end{array}$ & Groupe II (30-40 ans): $n=15$ \\
\hline Stade & Localisé : 2 & $\begin{array}{l}\text { Localement avancé : } 10 \\
\text { Métastatique : } 5\end{array}$ \\
\hline Traitement & Chirurgie curative : 2 & $\begin{array}{l}\text { Chirurgie curative : } 8 \\
\text { Traitement palliatif : } 7\end{array}$ \\
\hline
\end{tabular}

une prédisposition héréditaire dans la survenue des tumeurs vésicales chez le sujet jeune, et des mutations génétiques survenant sur les chromosomes 7 et 17 sont incriminées, mais ces résultats sont encore préliminaires ${ }^{12-15}$.

Dans cette étude rétrospective, nous avons évalué le profil évolutif des CCT de la vessie chez le jeune adulte. Nos résultats sont conformes à la littérature concernant la présentation clinique et les facteurs de risque, qui sont similaires quelle que soit la tranche d'âge concernée ${ }^{1,4,5,7,11}$. Cependant, les différentes séries ont des résultats divergents concernant les proportions des TVNIM et TVIM, ainsi que le profil évolutif de ces tumeurs (récidive, progression, rapidité d'évolution).

Certains auteurs ne notent pas de différence chez le sujet jeune. Aboutaieb ${ }^{4}$ a noté la présence de 14 TVIM chez 25 sujets âgés de moins de 40 ans. Johnson et Hillis $^{16}$ ont noté la présence de six TVIM sur une série de 22 jeunes adultes. Yossepowitch ${ }^{11}$ a noté la présence de 13 TVIM chez 74 patients de moins de 40 ans et n'a mis en évidence aucune différence significative dans la récidive et la progression des TVNIM en comparant deux groupes de patients âgés de moins de 40 ans et de plus de 65 ans.

Cependant, la majorité des séries rapportent un meilleur pronostic des CCT de la vessie chez le jeune adulte de moins de 40 ans et particulièrement avant 30 ans ${ }^{1,3,5,6,}$ ${ }^{17-21}$. Blanchard ${ }^{1}$ a constaté la présence de 22 TVNIM sur une série de 26 cas, dont 21 de stade Ta. L'évolution était meilleure avant 30 ans avec un taux de récidive de $38 \%$ contre $62 \%$ pour les sujets âgés de 30 à 40 ans. Fitzpatrick et Reda ${ }^{9}$ ont noté la présence de 43 TVNIM chez 50 patients âgés de moins de 40 ans. Le taux de récidive avant 30 ans était de $8 \%$, contre $54 \%$ entre 30 et 40 ans. Sur une série de 156 patients de moins de 40 ans, Erozenci ${ }^{17}$ a noté que la tumeur était superficielle dans $89,1 \%$ des cas. Le taux de récidive était de 10,3\% pour les tumeurs de stade Ta et de $38,4 \%$ pour les tumeurs de stade $\mathrm{T} 1$. Cette récidive est survenue chez 7,5\% des patients de moins de 30 ans et chez $22 \%$ des patients âgés entre 30 et 40 ans. Kutarski ${ }^{5}$ n'a noté aucune TVIM chez 20 patients de moins de 40 ans. Minciu ${ }^{21}$ n'a noté aucune TVIM chez sept patients de moins de 40 ans.

Ainsi, la majorité des auteurs s'accordent à dire que la tumeur du sujet de moins de 30 ans est typiquement superficielle, monofocale, de petite taille et dotée $d^{\prime}$ un faible taux de récidive ${ }^{1,5,6,17-19}$. L'aspect évolutif des TVNIM survenant entre 30 et 40 ans tend à se rapprocher de celui qui est noté chez les sujets âgés.

Pour les TVIM, le pronostic est identique à celui du sujet âgé et dépend du stade histologique et de l'évolution générale de la maladie au moment du diagnostic ${ }^{7,11}$. Cependant, certains auteurs ont rapporté une proportion élevée de TVIM et surtout un taux important de tumeurs déjà avancées au moment du diagnostic, ce qui laisse supposer un potentiel évolutif particulier des TVIM chez le sujet jeune. C'est ainsi qu'Aboutaieb $^{4}$ a rapporté $33 \%$ de tumeurs T4 au moment du diagnostic et dont l'évolution a été rapidement fatale. Pour Blanchard ${ }^{1}$, les TVIM représentent $13 \%$ de l'ensemble des tumeurs du sujet jeune et sont typiquement évoluées au moment du diagnostic (61\% de stade $\geq$ T3).

Dans notre série, les patients de moins de 30 ans avaient une TVNIM dans 13 cas $(86,6 \%)$, principalement monofocale, bien différenciée et de petite taille. Une récidive a été notée dans deux cas (15,3\%) sans progression quant au stade ou au grade. Pour les patients âgés de 30 à 40 ans, le profil des tumeurs se rapprochait de celui de la population âgée : la tumeur était une TVNIM dans 24 cas $(61,5 \%)$ et une TVIM dans 15 cas (38,5\%). Les TVNIM étaient moyennement différenciées dans plus de la moitié des cas ( 14 cas de tumeurs de grade II) et multifocale dans huit cas (33,3\%). Une récidive était notée dans huit cas $(33,3 \%)$ avec progression quant au stade dans deux cas (25\%).

Les TVIM ont représenté $31,5 \%$ de I'ensemble des tumeurs dans les deux groupes d'âge, ce qui représente un taux relativement élevé par rapport à la plupart des séries. Ces tumeurs étaient principalement agressives (localement avancées dans dix cas et métastatiques d'emblée dans cinq cas). Dans sept cas, la tumeur était jugée inopérable et le décès est survenu dans un délai moyen de 10 mois.

\section{Conclusion}

Le profil évolutif et le pronostic des CCT de la vessie chez le sujet jeune ont semblé dépendre de l'âge et du stade anatomique. Les TVNIM étaient principalement de stade Ta, bien différenciées, unifocales et peu récidivantes. À l'inverse, les TVIM étaient le plus souvent agressives. L'âge charnière semblait être 30 ans. Avant 30 ans, I'évolution des TVNIM était souvent favorable et au-delà de 30 ans, le pronostic semblait rejoindre celui du sujet âgé.

Intérêts conflictuels : Les auteurs affirment ne pas avoir d'intérêts conflictuels d'ordre financier ou personnel.

Cet article a été révisé par un comité de lecture. 


\section{Références}

1. Blanchard JM, Graziana JP, Bonnal JL et al. Bladder tumour in young patients:A series of 26 cases. Comparison with a review of the literature. Prog Urol 2003;13:227-33.

2. Fine SW, Humphrey PA, Dehner LP et al. Urothelial neoplasm in patients 20 years or younger: A clinicopathological analysis using the World Health Organization 2004 bladder consensus classification. J Urol 2005;174:1976-80. http://dx.doi.org/10.1097/01.ju.0000176801.16827.82

3. Kostakopoulos A, Stavropoulos N, Gavras P et al. Transitional cell carcinoma of the bladder in patients under 25 years of age. Prog Urol 1993;3:618-20.

4. Aboutaieb R, Dakir M, Sarrf I, et al. Bladder tumours in young patients. Prog Urol 1998;8:43-6.

5. Kutarski PW, Padwell A. Transitional cell carcinoma of the bladder in young adults. Br J Urol 1993;72:74955. http://dx.doi.org/10.1111/i.1464-410X.1993.tb16261.x

6. Migaldi M, Rossi G, Maiorana A et al. Superficial papillary urothelial carcinomas in young and elderly patients: A comparative study. BJU Int 2004;94:311-6. http://dx.doi.org/10.1111/i.1464410X.2004.04929.X

7. Ozbey I, Aksoy Y, Bicgi 0 et al. Transitional cell carcinoma of the bladder in patients under 40 years of age. Int Urol Nephrol 1999;31:655-9. http://dx.doi.org/10.1023/A:1007160522033

8. Chang SY, Ma CP. Transitional cell carcinoma of the urinary bladder in patients under 40 years of age. $\mathrm{Nr}$ J Urol 1987;60:343-4. http://dx.doi.org/10.1111/i.1464-410X.1987.tb04981.x

9. Fitzpatrick JM, Reda M. Bladder carcinoma in patients 40 years old or less. J Urol 1986; 135: 53-4.

10. Mc Carty JP, Gavrel GJ, Leblanc GA. Transitional cell carcinoma of the bladder in patients under 30 years of age. Urology 1979;13:487-9. http://dx.doi.org/10.1016/0090-4295(79)90453-9

11. Yossepowitch 0 , Dalbagni $G$. Transitional cell carcinoma of the bladder in young adults: Presentation, natura history and outcome. J Urol 2002;168:61-6. http://dx.doi.org/10.1016/S0022-5347(05)64832-X
12. Owen HC, Giedl J, Wild PJ et al. Low-frequency of epigenetic events in urothelial tumors in young patients. J Urol 2010;184:459-63 http://dx.doi.org/10.1016/i.juro.2010.03.131

13. Iori F, De Dominicis C, Liberti M et al. Superficial bladder tumours in patients under 40 years of age: Clinical, prognostic and cytogenetic aspects. Urol Int 2001;67:224-7. http://dx.doi.org/10.1159/000050992

14. Linn JF, Sesterhenn I, Mostofi FK et al. The molecular characteristics of bladder cancer in young patients. J Urol 1998;159:1493-6. http://dx.doi.org/10.1097/00005392-199805000-00022

15. Sidransky D, Von Eschenbach A, Tsai YC et al. Identification of p53 gene mutation in bladder cancer and urine samples. Science 1991;3:706-9. http://dx.doi.org/10.1126/science.2024123

16. Johnson DE, Hillis DS. Carcinoma of the bladder in patients less than 40 years old. J Urol 1978; 120:172-3.

17. Erozenci A, Ataus S, Pekyalcin A et al. Transitional cell carcinoma of the bladder in patients under 40 years of age. Int Urol Nephrol 1994;2:179-82. http://dx.doi.org/10.1007/BF02768283

18. Erpenbach $K$, Roth S, Rathert P. Therapy and followup of superficial bladder cancer in patients less than 30 years of age. Urologe A 1994;33:475-8.

19. Huguet Perez J, Errando Smet C et al. Transitional cell carcinoma of the bladder: Behaviour in young adults. Arch Esp Urol 1996;49:607-12.

20. Madrid Garcia FJ, Parra Muntaner L, Rivas Escudero JA et al. Transitional cell bladder carcinoma in patients younger than 40 years of age. Arch Esp Urol 1998;51:991-4.

21. Minciu R, Boiborean P, Daminescu L. Bladder tumours in young people. Urology 2009;74:S300. http:// dx.doi.org/10.1016/j.urology.2009.07.022

Correspondence: D' Ahmed Saadi, Service d'urologie, hôpital Charles-Nicolle, Tunis, Tunisie; dr.saadi.ahmed@gmail.com 